\title{
PROINFO e PROUCA: uma análise de dois programas oficiais que envolvem as
}

\section{multimídias}

\author{
PROINFO and PROUCA: an analysis of two official programs involving multimedia \\ PROINFO y PROUCA: un análisis de dos programas oficiales que involucran multimedia
}

Recebido: 02/12/2021 | Revisado: 09/12/2021 | Aceito: 21/12/2021 | Publicado: 03/01/2022

\author{
Mayara Rossi \\ ORCID: https://orcid.org/0000-0002-9182-1888 \\ Instituto Federal de Mato Grosso, Brasil \\ E-mail: professoramayararossi@hotmail.com \\ Geison Jader Mello \\ ORCID: https://orcid.org/0000-0002-0991-2327 \\ Instituto Federal de Mato Grosso, Brasil \\ E-mail: geison.mello@cba.ifmt.edu.br \\ Lilian Regina Simões \\ ORCID: https://orcid.org/0000-0002-7609-1813 \\ Secretaria de Estado de Educação, Brasil \\ E-mail: lilian.simoes@unemat.br
}

\begin{abstract}
Resumo
Este artigo tem como objetivo identificar os efeitos de dois programas oficiais que envolvem as multimídias: o ProInfro (Programa Nacional de Tecnologias na Educação) e o Prouca (Programa Um Computador por Aluno) na educação. O trabalho é baseado em uma pesquisa bibliográfica de natureza qualitativa, cuja análise de dados foi realizada segundo a análise de conteúdo de Bardin (2016). O período de levantamento e estudo se deu entre os meses de setembro a dezembro de 2019. O primeiro programa foi criado em 1997 para disseminar o uso pedagógico das tecnologias de informática e telecomunicações nas escolas. O segundo programa é mais recente, implantado em 2010 para promover a inclusão digital pedagógica e o desenvolvimento dos processos de ensino e aprendizagem. Como resultado podemos verificar que ambos os programas apresentam deficiência em sua execução: insuficiência na formação dos professores; falta de recursos financeiros para manutenção de laboratórios; infraestrutura inadequada; falta de assistência técnica; caráter instrumentalista e técnico e outros. Portanto, consideramos que somente oferecer as escolas equipamentos tecnológicos não se traduz a mudanças significativas no campo educacional. Desse modo, é necessário preparar os alunos para conviver na sociedade atual, por meio de programas ou projetos que promovam a inclusão de computadores, laptops, internet nas escolas, mas também através de uma formação de qualidade para o uso dos tais. Isso é exigido na contemporaneidade já que a tecnologia se faz presente em todos os aspectos da humanidade.
\end{abstract}

Palavras-chave: Multimídias; Tecnologias na educação; Programas oficiais.

\begin{abstract}
This article aims to identify the effects of two official programs involving multimedia: ProInfro (National Program for Technologies in Education) and Prouca (One Computer per Student Program) on education. The work is based on a bibliographical research of a qualitative nature, whose data analysis was carried out according to the content analysis of Bardin (2016). The survey and study period took place between September and December 2019. The first program was created in 1997 to disseminate the pedagogical use of computer and telecommunications technologies in schools. The second program is more recent, implemented in 2010 to promote pedagogical digital inclusion and the development of teaching and learning processes. As a result, we can see that both programs have a deficiency in their execution: insufficient teacher training; lack of financial resources for laboratory maintenance; inadequate infrastructure; lack of technical assistance; instrumentalist and technical character and others. Therefore, we consider that just offering schools technological equipment does not translate into significant changes in the educational field. Thus, it is necessary to prepare students to live in today's society, through programs or projects that promote the inclusion of computers, laptops, internet in schools, but also through quality training for the use of such. This is required nowadays, as technology is present in all aspects of humanity.
\end{abstract}

Keywords: Multimedia; Technologies in education; Official programs.

\section{Resumen}

Este artículo tiene como objetivo identificar los efectos de dos programas oficiales que involucran multimedia: ProInfro (Programa Nacional de Tecnologías en Educación) y Prouca (Programa Una Computadora por Estudiante) 
en la educación. El trabajo se basa en una investigación bibliográfica de carácter cualitativo, cuyo análisis de datos se realizó de acuerdo con el análisis de contenido de Bardin (2016). El período de encuesta y estudio se llevó a cabo entre septiembre y diciembre de 2019. El primer programa se creó en 1997 para difundir el uso pedagógico de las tecnologías de la computación y las telecomunicaciones en las escuelas. El segundo programa es más reciente, implementado en 2010 para promover la inclusión digital pedagógica y el desarrollo de procesos de enseñanza y aprendizaje. Como resultado, podemos verificar que ambos programas tienen deficiencias en su ejecución: insuficiente formación docente; falta de recursos económicos para el mantenimiento del laboratorio; infraestructura inadecuada; falta de asistencia técnica; De carácter instrumentalista y técnico y otros. Por tanto, consideramos que la simple oferta de equipamiento tecnológico a las escuelas no se traduce en cambios significativos en el ámbito educativo. Así, es necesario preparar a los estudiantes para vivir en la sociedad actual, a través de programas o proyectos que promuevan la inclusión de computadoras, laptops, internet en las escuelas, pero también a través de una formación de calidad para el uso de los mismos. Esto es necesario hoy en día, ya que la tecnología está presente en todos los aspectos de la humanidad.

Palabras clave: Multimedia; Tecnologías en educación; Programas oficiales.

\section{Introdução}

A partir de 1997, o Brasil estabeleceu uma política de tecnologias na educação, a qual pode ser traduzida no Programa Nacional de Tecnologias na Educação (ProInfo) e mais adiante, o Programa Um Computador por Aluno (Prouca). Desde então, trabalhos foram desenvolvidos tendo como foco esses programas (Basniak \& Soares, 2016). Assim, buscamos nessa pesquisa identificar os efeitos de dois programas oficiais que envolvem as multimídias: o ProInfro (Programa Nacional de Tecnologias na Educação) e o Prouca (Programa Um Computador por Aluno) na educação.

A motivação para o desenvolvimento desta pesquisa surgiu a partir da disciplina Tecnologias Aplicadas Ao Ensino de Ciências da Natureza e Matemática, do curso de Especialização em Ensino de Ciências da Natureza e Matemática da turma de 2019, do Instituto Federal de Mato Grosso, campus Juína.

Importante ressaltar que a tecnologia está presente em todos os lugares, e cada vez resolvemos mais problemas conectados, sendo assim, oferecer uma educação tecnológica de qualidade pode contribuir de forma significativa para o ensino e aprendizagem. O conviver virtual vai tornar-se quase tão importante como o conviver presencial (Moran, 2013). Por isso torna-se necessário fazer uma reflexão a respeito desses programas e entender seus contributos, já que eles envolvem a tecnologia, sendo está a justificativa deste trabalho.

Surgindo assim a problemática a ser respondida ao final desta pesquisa: Quais os efeitos positivos e negativos dos programas oficiais ProInfo e Prouca para a educação brasileira?

Nesse sentido, o presente trabalho está dividido estruturalmente dessa forma: primeiramente trazemos os conceitos introdutórios em relação à palavra multimídia, que pode ser definida como programas e sistemas de comunicação que se dá através de diferentes caminhos e meios: áudio, imagem, textos, etc.

Em seguida têm-se uma breve digressão histórica de programas oficiais envolvendo as multimídias, onde o marco das discussões sobre o uso da multimídia na educação, no Brasil, iniciou em um seminário sobre o uso de computadores no ensino em São Paulo, no ano de 1971. Ainda nesta seção veremos vários programas e projetos que foram desenvolvidos ao longo dos anos, a partir de 1971, todos com objetivo de inserir as chamadas multimídias nas escolas. Sendo os programas e projetos trazidos - I CONTECE, EDUCOM, CIED, FORMAR I e II; PRONINFE; PROJETO UCA; PBLE; TV ESCOLA; JORNAL DO PROFESSOR (SALTO PARA O FUTURO), PROINFRO e o PROUCA.

Depois é discutido em relação aos programas oficias que envolvem as multimídias, o ProInfro que significa Programa Nacional de Tecnologias na Educação, doravante ProInfo e o Prouca que significa Programa Um Computador por Aluno, que também a partir desse momento passa a se denominado apenas de Prouca.

Em seguida é descrita a metodologia utilizada para a elaboração do trabalho em voga, que como já citada no resumo, foi realizada uma pesquisa bibliográfica com abordagem qualitativa. 
Por fim, têm-se as considerações finais, onde encontra-se conclusões acerca dos resultados obtidos, em síntese, podemos perceber que há deficiências nos programas apresentados, sendo o principal "caráter instrumental e técnico", não explorando a potencialidade pedagógica dos meios tecnológicos.

Constituem-se como referências bibliográficas cujo deram sustentação e fundamentação teórica a este artigo as seguintes: Andriola e Gomes (2017); Basniak e Soares (2016); Bardin (2016); Barra (2017); Barros (2001); Borba e Lacerda (2015); Borges e Santos (2008); Brasil (2010); Brasil (2017); Carbonari (2001); Cotrim (2002); Fabris e Finco (2012); Figueiredo (2020); Lakatos e Marconi(2003); Maranhão (2001); Marques (2019); Medrado (2003); Monteiro (2005); Moran (2013); Oliveira (2001); Oliveira (2016); Silva e Souto (2018); Straub (2002); Valente (2011) e Xavier (2011) e sites TELECO, FNDE e MEC.

\section{Metodologia}

A investigação aqui descrita pode ser classificada como uma pesquisa bibliográfica de abordagem qualitativa.

Segundo Lakatos e Marconi (2003) a pesquisa bibliográfica, também conhecida como fontes secundárias abrange todo trabalho já tornado público em relação ao tema de estudo, incluindo publicações avulsas, livros, revistas, monografias, teses, material cartográfico, pesquisas, boletins, jornais, entre outros. Até mesmo meios de comunicações orais: rádio, gravações, filmes e televisão. "Sua finalidade é colocar o pesquisador em contato direto com tudo o que foi escrito, dito ou filmado sobre determinado assunto, inclusive conferências seguidas de debates que tenham sido transcritos por alguma forma, quer publicadas, quer gravadas" (Lakatos \& Marconi, 2003, p.183).

Oliveira (2016) classifica o enfoque qualitativo como analítico e reflexivo acerca do que é investigado, considerando sua realidade e o processo investigativo e não o resultado final em números.

De acordo com Bodgan e Biklen (1994) a realidade é enraizada nas percepções dos sujeitos, cujo intuito é interpretar e compreender significados através de narrativas e de observações no lugar de quantificações.

A investigação qualitativa normalmente ocorre em situações naturais e apresenta múltiplas características, podendo citar: usa múltiplos métodos de recolha de dados; há participação ativa do pesquisador; as questões a serem investigadas podem ser modificadas e redefinidas durante o desenvolvimento da pesquisa; é profundamente interpretativa e descritiva; sendo também indutiva; o investigador analisa os dados indutivamente; não havendo a preocupação em arranjar dados ou evidência para provar. Dessa forma, o investigador que utiliza a abordagem qualitativa preocupa-se mais com o processo e o contexto do que simplesmente com os resultados em si (Bodgan \& Biklen, 1994).

As buscas dos trabalhos para leitura e análise se deram a partir das palavras-chaves: programas oficias; multimídias e tecnologia na educação. Foram utilizados artigos científicos e dissertações de mestrado publicados em revistas científicas e divulgados no Periódico Capes, bem como em congressos na área da educação para a revisão bibliográfica. O levantamento e os momentos de estudos perduraram os meses de setembro a dezembro do ano de 2019.

Para a análise dos dados coletados, adotou-se a análise de conteúdo de Laurence Bardin (2016), um método de pesquisa que busca interpretar e compreender os significados das mensagens recebidas e escrita de textos, também utilizada para discursos diversos.

\section{Fundamentação Teórica}

O termo multimídia é utilizado a muito tempo, mesmo antes de surgir os computadores e demais tecnologias. Desse modo, para uma maior compreensão do conteúdo aqui desenvolvido é extremamente importante entender primeiramente o conceito de multimídia: 
Programas e sistemas em que a comunicação entre homem e computador se dá através de múltiplos meios de representação de informação (p.ex.: áudio, imagem estática, animação, gráficos e texto), ou seja, multimídia é o conjunto de recursos que visam estimular todos os sentidos, porém, os mais usuais são a visão e a audição. Multimídia significa que uma informação digital pode ser representada através de áudio, vídeo e animação em conjunto com mídias tradicionais (texto, gráficos e imagens) simultaneamente (Figueiredo, 2020, p. 03).

Portanto, podemos entender a multimídia como uma reunião de várias mídias. Essas podem se classificar em quatro categorias que são percepção, representação, armazenamento e transmissão. Abaixo segue o significado de cada uma dessas categorias, ainda segundo o autor supracitado:

Mídia de Percepção: São os equipamentos que têm como função estimular os sentidos dos seres humanos. A visão e a audição são os estímulos naturais provenientes de monitores e placas de som, por exemplo. O tato pode estar ligado a aplicações de realidade virtual. Existem estudos e protótipos já desenvolvidos para estímulo do olfato e paladar.

Mídia de Representação: São os elementos utilizados para representar uma idéia, como por exemplo: texto, imagem gráfica vetorial e estática (matricial), áudio, vídeo e animações.

Mídia de Armazenamento: São todos os meios que podem ser utilizados para o armazenamento de elementos da mídia de representação. Podem-se citar várias formas de armazenamento tais como: cartuchos para videogames, CDROM para computadores, discolaser interativos entre outras.

Mídia de Transmissão: São todos os meios de transmissão que são utilizados para veiculação da mídia de representação. A principal característica que deve ser levada em consideração é a largura de banda (bandwidth) que pode variar de algumas centenas de kbytes por segundo até algumas dezenas de Mbytes por segundo. Deve ser levado em conta, que estas aplicações devem ser processadas em tempo real e os dados têm uma natureza contínua (Figueiredo, 2020, p. 03 e 04).

Dessa maneira verifica-se que a multimídia foi um marco da evolução da tecnologia da informação. Era um termo pouco utilizado antigamente, diferentemente de hoje, pois o uso da multimídia se tornou indispensável nos sistemas atuais modernos. Atualmente a multimídia e a tecnologia estão presentes em praticamente todos os lugares, podendo citar entre eles os comércios em geral, nas escolas, nos lares, nos hospitais, nas mãos, bolsas e bolsos das pessoas que vivem na sociedade.

Segundo Moran (2013) a internet, as redes, o celular ou as denominadas multimídias estão revolucionando nossa vida no cotidiano. Essas tecnologias servem como apoio ou meio para inúmeras atividades diárias. Permite que um indivíduo aprenda sobre determinado assunto de distintas formas, em diferentes lugares e com espaços mais diminutos ainda. Sem a necessidade de estar sempre juntos em um espaço como a sala de aula para que isso aconteça.

Nesse momento adentrando a um breve histórico sobre programas oficiais envolvendo as multimídias para Silva e Souto (2018) apud Valente (1999) o marco de estudos e discussões sobre o uso da multimídia na educação, no Brasil, foi a partir de um seminário intensivo sobre o uso de computadores no ensino de Física, em 1971, na Universidade Federal de São Carlos em São Paulo. Nessa época, as iniciativas de incluir as tecnologias de informação na educação, no Brasil, se deram pela influência de outros países estrangeiros. Nesse período, o mundo vivenciava os conflitos finais da guerra fria.

Desde então, aconteceram outros eventos acadêmicos, em prol da inserção de computadores, nos processos de ensino e de aprendizagem, tal como: Primeira Conferência Nacional de Tecnologia em Educação Aplicada ao Ensino Superior (I CONTECE), onde professores e alunos realizaram experimentos, através de simulação de fenômenos de física (Borba \& Lacerda, 2015).

O primeiro projeto a ser lançado para implantação dos computadores no sistema público de ensino do Brasil foi o projeto Computador na Educação (EDUCOM), que tinha como finalidade introduzir o uso da tecnologia de informática nas escolas (Borba e Lacerda, 2015). Este não foi suficiente para efetivar o uso de computadores nas práticas escolares, porém deu continuidade a outros programas, como o Formar I e II e os Centros de Informática Educacionais (CIED). Conforme Valente (1999) o FORMAR I teve como objetivo a formação de professores e o FORMAR II a implantação dos centros nas escolas técnicas federais (CIET) ou no Ensino Superior (CIES). 
Em 1989, o MEC criou o Programa Nacional de Informática Educativa (PRONINFE). De acordo com dados encontrados no site Teleco, este veio com a proposta de que através do desenvolvimento de atividades e projetos articulados e convergentes, apoiados em fundamentação pedagógica sólida e atualizada, para assegurar a unidade política, técnica e científica imprescindível ao êxito dos esforços e investimentos envolvidos. Na década de 90, houve mais iniciativas por parte do governo federal, que junto com o MEC, implantaram diversos programas educativos voltados para a tecnologia. Em 1991 foi criado o Jornal do Professor, um programa de televisão que um ano mais tarde passou a ser chamado de salto para o futuro (Borba \& Lacerda, 2015).

Em 1996, o MEC criou um canal de televisão chamado TV Escola a fim de expandir e fortalecer a política de formação continuada aos educadores e junto com o salto para o futuro ofereceram kits tecnológicos às escolas. Mas nem todas as instituições de ensino foram contempladas por essa política. Todavia, em 2006 com o projeto DVD escola contemplaram as escolas que não haviam ganhado o kit. Em 1997 foi criado o Programa Nacional de Informática na Educação (PROINFO), no qual discutiremos a cerca dele na próxima seção com maiores detalhes (Borba \& Lacerda, 2015).

Em 2008, o governo federal criou o Programa Banda Larga na Escola-PBLE com o objetivo de conectar todas as instituições públicas à internet, rede mundial de computadores, por meio de tecnologias que propiciem qualidade e velocidade para inovar e melhorar o ensino público no Brasil. Após a implantação da banda larga, foi lançado o Projeto UCA com o intuito de intensificar o uso das tecnologias nas práticas pedagógicas dos professores (Borba \& Lacerda, 2015).

Em 2010 foi sancionado o Programa Um Computador por Aluno - PROUCA e o Regime Especial de Incentivo a Computadores para Uso Educacional - REICOMP, que será tema da próxima seção.

Nesse sentido, vimos que o governo federal juntamente com o MEC fez o bastante para levar a inserção das tecnologias digitais dentro das escolas, assim como tiveram grandes avanços durante esses vinte anos, porém, percebe-se que ainda há muito a avançar nessa área.

Adiante analisaremos dois programas oficiais que envolvem as multimídias na educação, significado, criação, objetivo, efeitos positivos e negativos e seus resultados: "PROINFO" e "PROUCA".

\section{Resultados e Discussão}

Ao analisar os programas trazidos em pauta iniciamos as discussões pelo PROINFO INTEGRADO "Programa Nacional de Tecnologia na Educação".

A implantação do Programa Nacional de Informática na Educação (ProInfo) se deu por meio de regime de colaboração entre os entes federados da União, Estados e Municípios em 9 de abril de 1997 pela Portaria nº 522/MEC. Em seu primeiro artigo dispõe sobre sua finalidade: “disseminar o uso pedagógico das tecnologias de informática e telecomunicações nas escolas públicas de Ensino Fundamental e médio pertencentes às redes estadual e municipal” (Basniak \& Soares, 2016, p. 202).

Em seu artigo primeiro - Parágrafo único - consta os objetivos do ProInfo:

I - Promover o uso pedagógico das tecnologias de informação e comunicação nas escolas de educação básica das redes públicas de ensino urbanas e rurais;

II - Fomentar a melhoria do processo de ensino e aprendizagem com o uso das tecnologias de informação e comunicação;

III - Promover a capacitação dos agentes educacionais envolvidos nas ações do Programa;

IV - Contribuir com a inclusão digital por meio da ampliação do acesso a computadores, da conexão à rede mundial de computadores e de outras tecnologias digitais, beneficiando a comunidade escolar e a população próxima às escolas;

V - Contribuir para a preparação dos jovens e adultos para o mercado de trabalho por meio do uso das tecnologias de informação e comunicação; e

VI - Fomentar a produção nacional de conteúdos digitais educacionais (Brasil, 2007, p. 01). 
Segundo Basniak e Soares (2016) o programa prevê a implantação de equipamentos tecnológicos nas escolas, onde o Ministério da Educação fica responsável por comprar, distribuir e instalar laboratórios de informática nas escolas públicas brasileiras de ensino básico. Mas, para que isso ocorra, Estados, Distrito Federal e Municípios devem garantir a estrutura adequada para receber os laboratórios e capacitar os educadores para uso das máquinas e tecnologias. Portanto, o programa leva às escolas computadores, recursos digitais e conteúdos educacionais. Ainda para os autores supracitados:

O programa foi vinculado à Secretaria de Educação a Distância (SEED), do MEC, em articulação com as secretarias de educação do Distrito Federal, dos Estados e dos Municípios. Implantou, até o final de 1998, 119 Núcleos de Tecnologia Educacional (NTEs) em 27 Estados e no Distrito Federal; e capacitou, por intermédio de cursos de especialização em Informática em Educação (360 horas), cerca de 1.420 multiplicadores para atuarem nos NTEs.

A partir de 2007, o ProInfo passou a ser denominado Programa Nacional de Tecnologia Educacional, sendo redefinido como um programa educacional que busca promover o uso pedagógico da informática na rede pública de educação básica (Basniak \& Soares, 2016, p. 202).

Nessa perspectiva, vimos que no ano de 2017 o ProInfro passou a ser denominado Programa Nacional de Tecnologia na Educação - PROINFO Integrado. Ao implementar novas ações, o PROINFO passou a ser chamado Programa Nacional de Tecnologia na Educação - PROINFO Integrado e não mais apenas de PROINFRO. Nesse intento, por meio do PROINFO Integrado, o MEC ofertou três cursos de formação continuada para professores sobre Introdução à educação digital. O primeiro curso consistiu basicamente sobre instruções ao sistema operacional Linux, bem como de recursos básicos da internet aos gestores e docentes que ainda não possuíam domínio nessas áreas. O segundo cursou enfatizou a utilização pedagógica das tecnologias digitais nos processos de ensino e aprendizagem. Por fim, o terceiro curso salientou sobre a importância do uso das TD em projetos de ensino (Silva \& Souto, 2019).

Para fazer parte do Proinfo Urbano e/ou Rural, o município deveria seguir três passos: adesão, o cadastro e a seleção das escolas. A adesão é o compromisso do município com as diretrizes do programa, imprescindível para o recebimento dos laboratórios. Após essa etapa, deveria ser feito o cadastro do prefeito no sistema, que permitiria o próximo passo, que é a inclusão das escolas no ProInfo.

Basniak e Soares (2016) colocam que os laboratórios do ProInfo são de dois formatos. Por um lado, tem-se o ProInfo Urbano e por outro lado o rural. O primeiro composto por "1 servidor de rede, 15 estações para o laboratório de informática, 2 estações para área administrativa, monitores LCD, 1 roteador wireless, 1 impressora a laser. O segundo composto por: 1 servidor, 4 estações, monitores LCD, 1 impressora a jato de tinta" (p. 202). Em suma, as estações eram compostas por multiterminais que consistiam em ligar vários monitores, mouses e teclados em apenas um único terminal.

Sobre o PROINFO Integrado Straub (2002) acrescenta que houve insuficiência na qualificação dos profissionais da escola envolvidos no Programa; descumprimento do cronograma de implementação e disponibilização da infraestrutura prevista. Para Barros (2001) foram visíveis as precárias condições básicas das escolas e conforme Carboni (2001) pouco despreparo dos professores e insuficiência de verbas.

Cotrim (2002) encontrou deficiência na formação de professores durante o programa; falta de conexão com a Internet; a dinâmica das escolas pouco se alterou; falta de recursos financeiros para a manutenção dos laboratórios; computador tido mais como meio de motivação e instrumento de memorização de conteúdos.

Medrado (2003) cita a fragmentação da inserção e utilização dos recursos da informática no interior das escolas; falta de capacitação dos professores; infraestrutura inadequada; falta de assistência técnica; dificuldade de acesso às salas de informática pelos alunos.

Monteiro (2005) aponta que os computadores foram utilizados fora do período das aulas em atividades não pedagógicas; pequeno interesse dos professores no uso dos laboratórios; pouca adesão nas unidades escolares a todas as propostas indicadas nos objetivos do ProInfo. 
Oliveira (2011) diz que é limitado o alcance dos recursos do ProInfo. Enfim, para Maranhão (2001) o curso de capacitação oferecido pelo ProInfo aos professores foi insuficiente.

Em relação ao PROUCA "Programa Um Computador por Aluno", encontramos dados no site FNDE que descrevem este programa como um registro de preços (RPN) do FNDE para que os estados e municípios pudessem comprar com recursos próprios ou com financiamento do BNDES.

O Prouca foi instituído pela Lei $\mathrm{n}^{\mathrm{o}} 12.249$, de 14 de junho de 2010, com o objetivo promover a inclusão digital pedagógica e o desenvolvimento dos processos de ensino e aprendizagem de alunos e professores das escolas públicas brasileiras, mediante a utilização de computadores portáteis denominados laptops educacionais:

Art. $1^{\circ}$ Este Decreto regulamenta o Programa Um Computador por Aluno - PROUCA e o Regime Especial de Incentivo a Computadores para Uso Educacional - REICOMP.

$\S 1^{\circ}$ O PROUCA tem o objetivo de promover a inclusão digital nas escolas das redes públicas de ensino federal, estadual, distrital, municipal e nas escolas sem fins lucrativos de atendimento a pessoas com deficiência, mediante a aquisição e a utilização de soluções de informática, constituídas de equipamentos de informática, de programas de computador - software - neles instalados e de suporte e assistência técnica necessários ao seu funcionamento (Brasil, 2010, p. 01).

Os equipamentos citados continham um sistema operacional específico e características físicas que facilitam o uso e garantia de segurança dos estudantes, que foi desenvolvido especialmente para uso no ambiente escolar. O FNDE facilita a aquisição desses equipamentos com recursos dos próprios estados e municípios por meio da adesão ao pregão eletrônico.

Conforme Andriola e Gomes (2017) apoiados em Fabris e Finco (2012), além de Borges e Santos (2008) enfatizam que o Prouca teve como objetivo o seguinte descrito:

Ser um projeto educacional de uso da TDIC como forma de promover inovações pedagógicas no ensino, mas também promover a inclusão digital. Outra meta do programa é proporcionar o adensamento da cadeia produtiva comercial no Brasil. A ideia de implantação desse programa no Brasil está relacionada com as iniciativas da organização não governamental internacional One Laptop per Child (OLPC), criada em 2005. Trata-se de um projeto promovido por uma fundação sem fins lucrativos, que se propõe desenvolver, produzir e distribuir laptops de baixo custo, com configurações diferenciadas e específicas, a alunos de países em desenvolvimento, para uso na modalidade 1:1, isto é, um computador para cada indivíduo (Andriola \& Gomes, 2017, p. 269).

Em 2007 alguns experimentos do Prouca foram realizados em escolas de São Paulo, assim como no Rio de Janeiro, Rio Grande do Sul, Tocantins, Distrito Federal, que envolveu acompanhamento e avaliação de várias universidades brasileiras. Entretanto, somente em 2010 foi criado institucionalmente o Prouca, como mencionado anteriormente.

O programa criou uma expectativa de transformações na cultura de produção e apropriação de saberes. Porém, para Andriola e Gomes (2017) em consonância com Valente (2011) não adianta somente implantar ou prover acesso aos computadores ou laptops, esse fato não vai alterar a maneira como a educação é desenvolvida.

Marques (2019) esboça que o programa foi desenvolvido com grande deficiência na formação dos professores para o uso das tecnologias de informação e comunicação.

Xavier (2011) afirma que foi o mais audacioso projeto de informatização da educação básica brasileira; impactos mais pela movimentação econômica no setor e aparelhamento tecnológico relativo das escolas do que a ocorrência de mudanças do paradigma educacional. Nesse intento, enquadra-se no contexto das demais políticas educacionais de informatização, sem representações significativas de mudanças abrangentes de práticas pedagógicas.

Enfim, o Prouca é apontado como um importante programa. Em contrapartida alguns trabalhos indicam que há necessidade de adequação das políticas públicas relacionadas as tecnologias nas escolas públicas, para que tenha continuidade 
e não mudem a cada novo governo, que também contenha uma infraestrutura adequada, com condições de manutenção (Basniak \& Soares, 2016).

\section{Considerações Finais}

Por meio da pesquisa foi possível verificar que através do ProInfo Integrado e do Prouca, ofertou-se cursos de formação continuada aos professores sobre Introdução à educação digital, Tecnologias na educação e temas relacionados a área em questão. Mas, ambos os programas não apresentaram resultados concretos em relação à inclusão social nas escolas públicas brasileiras.

Esse fato advém de inúmeros fatores: as condições físicas e estruturais das instituições escolares; dificuldade de acesso; inadequada formação dos professores para o uso dos recursos tecnológicos digitais, sendo a última o principal motivo. Desse modo, não vemos inovação nas salas de aula, tampouco aprendizagens efetivas com apoio das tecnologias, mas vê-se predominantemente um caráter técnico e instrucionista de equipamentos tecnológicos.

Houve descumprimento do cronograma de implementação do ProInfo e da disponibilização da infraestrutura prevista. Também as formações dos professores previstas pela falta de recursos e formadores.

Nesse sentido, mesmo que o ProInfo tenha sido criado e implantado em 1997, ou seja, há vinte e quatro anos e tenha contribuído para distribuir equipamentos em escolas, oferecido formações para professores e levado os mesmos a refletirem sobre os processos pedagógico, ainda não se efetivou a inclusão digital no cotidiano escolar.

Em relação ao Prouca, este foi desenvolvido com grande deficiência na formação dos professores e não houve representações significativas de mudanças abrangentes de práticas pedagógicas.

Portanto, nos dois programas os recursos tecnológicos não são tratados como instrumentos de ensino, enfatizando mais uma vez que prevalece o caráter instrucionista das aulas, sem aproveitar as grandes potencialidades pedagógicas que as tecnologias podem proporcionar e promover em sala de aula.

Nesse escopo, compreende-se nessa pesquisa que apenas disponibilizar equipamentos digitais as escolas não é garantia de melhoria na qualidade da educação. Enfim, concluímos que as políticas de tecnologias educacionais se encontram em caráter técnico e instrumentalista, necessitando serem revistas para que possamos avançar. Desse modo, é importante desenvolver outros trabalhos relacionados as tecnologias digitais e programas que envolvem multimídias, sendo este trabalho uma contribuição para futuras pesquisas a respeito do tema.

\section{Referências}

Abreu, M. H. T.; Nogueira, T. A.; Oliveira, V. A.; Matos, L. M. R.; Oliveira, B. G. S. \& Sousa, P. G. (2020). Investigações acerca da implantação do Programa Nacional de Melhoria do Acesso e da Qualidade da Atenção Básica (PMAQ) no Estado do Piauí. Research, Society and Development, 9(11), 1-19.

Andriola, W. B. \& Gomes, C. A. S. (2017). Programa Um Computador Por Aluno (PROUCA): uma análise bibliométrica. DOI: 10.1590/0104-4060.48230. Educar em Revista, 63, 267-288.

Bardin, L. Análise de conteúdo. (2016). Traduzido por Luís Antero Reto, Augusto Pinheiro. São Paulo: Edições 70.

Barra, A. S. B. (2007). O ProInfo e a formação de professores em Goiânia. Brasília, DF. Dissertação de Mestrado. Universidade de Brasília, 165 p.

Barros, F. G. R. (2001). O programa nacional de informática na educação no Estado de Goiás e suas implicações na educação escolar. Uberlândia, MG. Dissertação de Mestrado. Universidade Federal de Uberlândia, 198 p.

Basniak, M. I. \& Soares, M. T. C. (2016). O PROINFO e a disseminação da Tecnologia Educacional no Brasil. Educação Unisinos, $20(2), 201-214$.

Bogdan, R. \& Biklen, S. (1994). Investigação qualitativa em educação. Porto: Porto Editora.

Borba, M.C. \& Lacerda, H. D. G. (2015). Políticas Educacionais e Tecnologias Digitais: um celular por aluno. Revista Educação Matemática Pesquisa (Online), 17, 490- 507. 
Borges, M. K. \& Santos, M. B. F. (2008). Um retrospecto histórico do projeto OLPC no Brasil e no mundo. In: seminário de pesquisa em educação da região sul - anped sul, 7. Anais... Itajaí, SC.

Brasil. (2007). Decreto 6.300 de 12 de dezembro de 2007 - Programa Nacional de Tecnologia Educacional- ProInfo. https://www.planalto.gov.br/ccivil_03/_ato2007-2010/2007/decreto/d6300.htm.

Brasil. (2010). Decreto 7.750 de 10 junho de 2010. Programa um computador por aluno. http://www.planalto.gov.br/ccivil_03/_ato20112014/2012/decreto/d7750.htm.

Carbonari, G. (2001). O programa nacional de informática na educação nas escolas públicas de Ijuí/RS. Ijuí, RS. Dissertação de Mestrado. Universidade Regional do Noroeste do Estado do Rio Grande do Sul, 189 p.

Cotrim, E. C. (2002). O ProInfo na escola - as contradições da modernidade: um estudo da implementação do programa no município de Silvânia-Goiás. Goiânia, GO. Dissertação de Mestrado. Pontifícia Universidade Católica de Goiás, 132 p.

Dantas, D. M. P.; Cristovam, F. K. G.; Araújo, M. J.; Brandão, I. A. \& Santana, A. M. S.; Pê, S. Z. (2020). O despacho da sala de aula e Tecnologias Digitais. Research, Society and Development, 9(11), 1-18.

Fabris, L. L.; Finco, M. D. (2012). Percepção de Escolares no Uso de Laptops Educacionais no Contexto do Projeto UCA. In: simpósio brasileiro de informática na educação, 23. Anais... (SBIE).

Figueiredo, R. T. (2020). Apostila de Sistemas Multimídia. Petrolina - PE. Disponível em: https://central3.to.gov.br/arquivo/453373/. Acesso em 26 dez. 2020. FNDE - Ministério da Educação. Um computador por aluno (PROUCA). https://www.fnde.gov.br/index.php/programas/proinfo/eixos-de-atuacao/programaum-computador-por-aluno-prouca.

Lakatos, E. V. \& Marconi, M. A. (2003). Fundamentos da metodologia científica. $5^{\circ}$ educação. Editora Atlas - São Paulo.

Maranhão, T.B.N. 2001. O professor e a nova tecnologia da informação - o computador: experiência do ProInfo no Piauí. Belo Horizonte, MG. Dissertação de Mestrado. Universidade Federal de Minas Gerais, 172 p.

Marques, A.C.C. 2019. O Projeto Um Computador por Aluno - UCA: reações na escola, professores, alunos, institucional. Curitiba, PR. Dissertação de Mestrado. Universidade Federal do Paraná, 85 p.

MEC - Ministério da Educação. ProInfro Apresentação. http://portal.mec.gov.br/proinfo.

Medrano, E.M. de O. (2003). O Programa Nacional de Informática na Educação - PROINFO: avaliação do impacto nas escolas estaduais do Ensino Médio em São Carlos. Araraquara, SP. Dissertação de Mestrado. Universidade Estadual Paulista Júlio de Mesquita Filho, 189 p.

Monteiro, V. da S. (2005). Informática e democratização do ensino: o uso de computadores na rede pública do município de Araraquara. Araraquara, SP. Dissertação de Mestrado. Universidade Estadual Paulista Júlio de Mesquita Filho, 132 p.

Moran, J. (2013). Educação e Tecnologias: Mudar para valer! Papirus, 21 a ed, p. 12-14 (com modificações).

Oliveira, H. Q. (2011). Tecnologias de informação e comunicação na educação e inclusão sócio-digital: uma avaliação do Programa de Informática na Educação - PROINFO em Fortaleza. Fortaleza, CE. Dissertação de Mestrado. Universidade Federal do Ceará, 143 p.

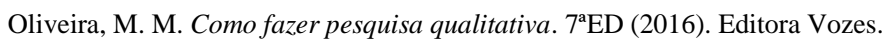

Pereira, N. V. \& Araújo, M. S. T. de. (2020). Uso de recursos tecnológicos na Educação: caminhos e perspectivas. Research, Society and Development, 9(8), $1-18$.

Silva, J. B. \& Souto, D. L. P. (2018). Tecnologias digitais: políticas da formação continuada ofertada pelo CEFAPRO aos professores da unidocência para o Ensino de Ciências. Revista ACTIO: Docência em Ensino.

Schossler, A. B.; Medeiros, L. M.; Paprosqui, J.; Germani, A. R. M. \& Folmer, I. (2021). Digital inclusion in field schools. Research, Society and Development, 10(5), 1-9.

Straub, S.L.W. (2002). O computador no interior da escola pública: avanços, desafios e perspectivas. Florianópolis, SC. Dissertação de Mestrado. Universidade Federal de Santa Catarina, 130 p.

Teleco. Inteligência em Comunicações. Interatividade Gráfica I: Tecnologia e Educação. https://www.teleco.com.br/tutoriais/tutorialintgraf1/pagina_3.asp.

Tessari, R. M.; Fernandes, C. T.; Campos, M. das G. (2020). O uso das mídias digitais na educação: da perspectiva para a prática. Research, Society and Development, $9(11), 1-24$.

Valente, J. A. Um laptop para cada aluno: promessas e resultados educacionais efetivos. In: Almeida, M. E. B. \& Prado, M. E. B. B. (Org.). (2011). O computador portátil na escola: mudanças e desafios nos processos de ensino e aprendizagem. São Paulo: Avercamp.

Valente, J. A. (1999). Informática na Educação no Brasil: análise e contextualização histórica. In: Valente, J. A. (Org.). O computador na sociedade do conhecimento. Campinas: Unicamp/NIED.

Xavier, L.G. de S. (2011). O “Programa Um Computador Por Aluno” - PROUCA e o ensino de Geografia. Rio de Janeiro, RJ. Dissertação de Mestrado. Universidade do Rio de Janeiro, 228 p. 